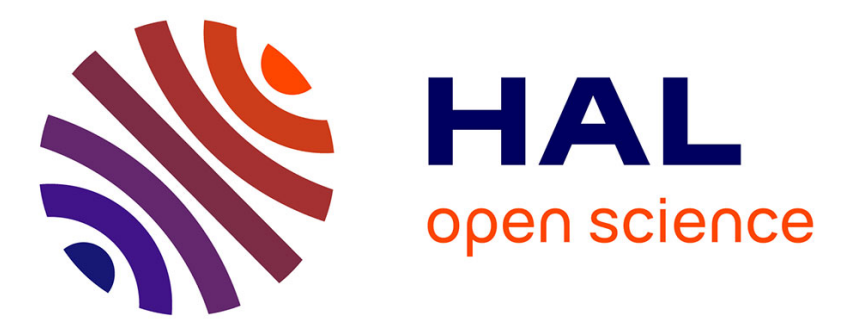

\title{
De la Russie à la Chine? Le basculement énergétique de l'Asie centrale
}

\author{
Julien Vercueil
}

\section{To cite this version:}

Julien Vercueil. De la Russie à la Chine? Le basculement énergétique de l'Asie centrale. Mondes en Développement, 2015, 169, pp.47 - 47. 10.3917/med.169.0047 . halshs-01422013

\section{HAL Id: halshs-01422013 \\ https://shs.hal.science/halshs-01422013}

Submitted on 23 Dec 2016

HAL is a multi-disciplinary open access archive for the deposit and dissemination of scientific research documents, whether they are published or not. The documents may come from teaching and research institutions in France or abroad, or from public or private research centers.
L'archive ouverte pluridisciplinaire HAL, est destinée au dépôt et à la diffusion de documents scientifiques de niveau recherche, publiés ou non, émanant des établissements d'enseignement et de recherche français ou étrangers, des laboratoires publics ou privés.

\section{(1) (1) $\$$}

Distributed under a Creative Commons Attribution - NonCommercial - NoDerivatives 44.0 


\title{
De la Russie à la Chine? \\ Le basculement énergétique de l'Asie centrale
}

\author{
Julien Vercueil \\ CREE, INALCO \\ CEMI, EHESS \\ julien.vercueil@inalco.fr
}

Résumé : l'Asie centrale est un voisinage partagé de la Chine et la Russie à forts enjeux pour ces deux grands émergents. La dynamique chinoise concurrence les influences russe et occidentale dans la région. L'article discute l'hypothèse d'un basculement énergétique de la région vers la Chine en étudiant l'évolution de quatre paramètres : le rapport aux compagnies occidentales, la capacité d'émancipation visà-vis des réseaux de transport d'hydrocarbures soviétiques, les stratégies des grandes compagnies russes et la dynamique des besoins des principaux clients de la région - en particulier la Chine.

Mots clés : Asie centrale, Russie, Chine, échanges internationaux, énergie.

Mots clés : Asie centrale, Russie, Chine, intégration économique régionale, énergie.

\section{From Russia to China? An energetic switch for central Asian countries}

\begin{abstract}
Central Asia is a shared neighbourhood for China and Russia. The Chinese dynamics is challenging Russian and western influences in the region. This article discusses the hypothesis of an energetic switch of the region toward China by studying four key parameters: the relationships with western firms, the ability of central Asian energy exporters to pass-by the soviet-time pipeline networks, Russian firms strategies and the dynamics of the main customers of regional mineral resources particularly China.
\end{abstract}

Keywords : Central Asia, Russia, China, external trade, energy.

Codes JEL : F15, O53, P33, Q35, Q48 
L'Asie centrale est une région enclavée dont le passé soviétique justifie qu'on la considère comme un ensemble économique, mais qui est aujourd'hui caractérisée par l'hétérogénéité des cinq pays qui la composent. L'économie dominante - le Kazakhstan - pèse deux tiers du PIB régional et possède un niveau de revenu par habitant largement supérieur à la moyenne régionale (Tableau 1). L'hétérogénéité de la région est aussi structurelle : le groupe est partagé entre pays exportateurs d'hydrocarbures (Kazakhstan, Turkménistan, Ouzbékistan) et pays importateurs (Tadjikistan et Kirghizstan). Partout, l'agriculture emploie plus d'un quart de la population active (la moitié au Tadjikistan et au Turkménistan). Cette part est aujourd'hui en déclin, au profit des activités industrielles et de services.

Tableau 1. Quelques indicateurs fondamentaux des pays d'Asie centrale

\begin{tabular}{|c|c|c|c|c|c|c|c|}
\hline & $\begin{array}{c}\text { Super- } \\
\text { ficie } \\
\mathbf{( k m 2 )}\end{array}$ & $\begin{array}{c}\text { Population } \\
\text { (Mio hab) }\end{array}$ & $\begin{array}{c}\text { PIB } \\
\mathbf{( M r d} \\
\text { US \$) }\end{array}$ & $\begin{array}{c}\text { PIB par } \\
\text { hab } \\
\mathbf{( \$ ~ P P A ) ~}\end{array}$ & $\begin{array}{c}\text { Rang } \\
\text { IDH } \\
\mathbf{( 1 8 6} \\
\text { pays) }\end{array}$ & $\begin{array}{c}\text { Part de la } \\
\text { population } \\
\text { sous le seuil } \\
\text { de 2 \$ PPA/j }\end{array}$ & $\begin{array}{c}\text { Croissance } \\
\text { moyenne } \\
\mathbf{( 2 0 1 0 - 2 0 1 3 )}\end{array}$ \\
\hline Kazakhstan & 2725 & 17 & 220 & 14391 & 70 & $0,8 \%$ & $6,4 \%$ \\
\hline Kirghizie & 200 & 5,6 & 7,2 & 2611 & 125 & $21,1 \%$ & $3,7 \%$ \\
\hline Ouzbékistan & 447 & 32 & 56,5 & 3762 & 116 & n. d. & $8,2 \%$ \\
\hline Tadjikistan & 143 & 8,1 & 8,5 & 2354 & 133 & $27,4 \%$ & $7,2 \%$ \\
\hline Turkménistan & 488 & 6,2 & 40,6 & 9510 & 103 & $49,7 \%$ & $11,3 \%$ \\
\hline Asie centrale & $\mathbf{4 0 0 3}$ & $\mathbf{6 8 , 9}$ & $\mathbf{3 3 2 , 8}$ & $\mathbf{6 6 4 3}$ & & & $\mathbf{7 , 3} \%$ \\
\hline Russie & 17098 & 143 & 2118 & 17884 & 57 & $0,3 \%$ & $3,4 \%$ \\
\hline Chine & 9598 & 1361 & 9181 & 10695 & 91 & $18,6 \%$ & $8,7 \%$ \\
\hline
\end{tabular}

Sources : IMF World Economic Outlook 2014, World Bank, PNUD.

Les ressources naturelles sont l'une des principales richesses de la zone. Si le Kazakhstan, aidé par l'immensité de son territoire, domine ses voisins pour les réserves de charbon, de pétrole et d'uranium (entre 87 et $94 \%$ du total des ressources prouvées), c'est le Turkménistan qui est le principal dépositaire de gaz $(86 \%)$ et le Tadjikistan qui a les plus fortes capacités hydroélectriques (44\%), plus équitablement partagées dans cette région soulevée sur ses marges orientales par les massifs du Pamir et du Tian Shan. Ensemble, ces pays ne représentent toutefois qu'une fraction modeste des ressources d'hydrocarbures ( $4 \%$ pour le pétrole, $34 \%$ pour le gaz) et des capacités hydroélectriques $(25 \%)$ de la Russie. En revanche, les gisements d'uranium du Kazakhstan dépassent en capacité ceux de la Russie (Tableau 2). En l'absence de réserves énergétiques prouvées significatives, le Tadjikistan et la Kirghizie doivent compter sur l'exportation d'autres ressources naturelles : l'or pour la Kirghizie (mine de Kumtor), l'aluminium et l'énergie hydroélectrique pour le Tadjikistan. L'équilibre de leur compte courant est assuré par les transferts de revenus en provenance des travailleurs expatriés, qui ont pu représenter certaines années près de la moitié du PIB de ces pays. 
Tableau2. Réserves et capacités énergétiques en Asie centrale

\begin{tabular}{|c|c|c|c|c|c|c|}
\hline & KAZAKHSTAN & KIRGHIZIE & OUZBEKISTAN & TADJIKISTAN & TURKMENISTAN & RUSSIE \\
\hline $\begin{array}{c}\text { Réserves de } \\
\text { charbon } \\
\text { (Mns tep) }\end{array}$ & 15900 & 385 & 902 & 178 & - & 74500 \\
\hline $\begin{array}{c}\text { Réserves de gaz } \\
\text { (Mns tep) }\end{array}$ & 2070 & 5 & 1580 & 5 & 21700 & 41100 \\
\hline $\begin{array}{c}\text { Réserves de } \\
\text { pétrole (Mns t) }\end{array}$ & 2910 & 5 & 80 & 2 & 83 & 12100 \\
\hline $\begin{array}{c}\text { Réserves } \\
\text { d'uranium (t) }\end{array}$ & 629 & - & 96,2 & - & - & 487,2 \\
\hline $\begin{array}{c}\text { Capacités } \\
\text { hydroélectriques } \\
\text { installées (GW) }\end{array}$ & 2,27 & 2,91 & 1,71 & 5,5 & 0,001 & 49,7 \\
\hline
\end{tabular}

Source: World Energy Council (www.worldenergy.org) données de 2011, dernière consultation 02/09/2014. Uranium: World Nuclear Association (www.world-nuclear.org), données 2012, dernière consultation 02/09/2014.

$M n s$ : millions; tep : tonnes équivalent pétrole; $t$ : tonnes; $G W$ : gigawatts.

En remettant en cause le système de relations inter-républicaines conçu dans le cadre de la planification centralisée, la dissolution de l'URSS a fait surgir plusieurs problèmes de coopération régionale (Pomfret, 1995, 2006). La rupture des liaisons héritées de la planification soviétique et les priorités géoéconomiques divergentes des autorités de ces pays nouvellement indépendants ont provoqué l'effondrement $(-80 \%)$ des échanges intra-régionaux durant la première moitié des années 1990 (Freinkman et alii, 2004).

Au Nord et à l'Est, Russie et Chine partagent les frontières les plus longues avec les pays de la région. A l'Ouest, la Mer caspienne, au Sud, l'Afghanistan et l'Iran, complètent le voisinage immédiat (Cartes 1 et 2). Leur proximité avec les émergents majeurs que sont la Russie et la Chine offre des opportunités économiques à ces pays. Mais les écarts de PIB et de revenu par habitant trahissent aussi des différences d'échelle et de productivité considérables avec ces grands voisins. Dans ces conditions, ce sont jusqu'ici les enjeux énergétiques qui ont dominé les grandes orientations géoéconomiques des pays de la région.

L'objectif de cet article est de discuter l'hypothèse d'un basculement énergétique de l'Asie centrale. Faute d'une dynamique effective d'intégration économique régionale, ce sont les relations avec l'extérieur qui sont à l'origine des principales transformations économiques en cours (1). Plus précisément, les rapports entretenus avec les compagnies occidentales, la recherche d'une moindre dépendance envers les réseaux hérités de la période soviétique pour l'exportation d'hydrocarbures, les capacités stratégiques des entreprises énergétiques russes et l'évolution des besoins des principaux clients de la région en matière énergétique sont quatre paramètres qui montrent que l'influence russe sur les pays d'Asie centrale est de plus en plus concurrencée, notamment par la Chine (2). A l'avenir, l'évolution de la demande énergétique chinoise et ses effets sur les besoins en importations du pays contribueront à déterminer l'ampleur de ce basculement (3).

\section{La faiblesse de l'intégration régionale a laissé le champ libre aux projets extra-régionaux}

Bien que les flux intra-régionaux de main-d'œuvre soient importants ${ }^{1}$, l'intégration commerciale de la région est faible (Pomfret, 2009, 2010a). La question énergétique, qui aurait pu constituer l'une des bases d'un éventuel projet d'intégration économique régionale, a plutôt conduit, dans un premier temps, à aggraver la fragmentation de la zone. Ainsi, lorsque le système de redistribution de l'énergie électrique de l'URSS est devenu caduc, des rivalités sont apparues pour l'accès aux ressources hydroélectriques

\footnotetext{
1 En 2014, les estimations officielles de la proportion de travailleurs migrants dans la population sont de 27 \% en Ouzbékistan, 18\% pour le Tadjikistan, $14 \%$ pour la Kirghizie (Organisation Internationale des Migrations, www.iom.int).
} 
régionales. Les pays des régions montagneuses (Kirghizie et Tadjikistan) où les capacités hydroélectriques sont les plus importantes n'ont pas su accorder leurs intérêts à ceux des régions des plaines (Ouzbékistan), qui dépendent d'une agriculture fortement consommatrice d'eau. Des tensions sur l'approvisionnement en électricité de l'Ouzbékistan par le Tadjikistan en 2007-2008, puis entre l'Ouzbékistan et la Kirghizie suite au projet de développement de barrages en Kirghizie, ont montré en creux l'absence de projet panrégional de gestion des ressources hydro-électriques, qui aurait permis de lier les pays par des solidarités institutionnalisées de fait.

Tous produits confondus, les échanges intra-régionaux ne dépassent pas $4 \%$ du total des exportations et $5,6 \%$ des importations (Tableau 3). Les seules catégories de produits pour lesquelles ces flux sont relativement importants sont les produits agricoles non transformés (céréales, farines, fruits et légumes). La faiblesse des échanges intra-régionaux accroît l'incidence de l'enclavement de la région, dont aucun pays n'a accès aux mers ouvertes.

Tableau3. Échanges commerciaux en Asie centrale post soviétique : Analyse par produits (2011-2013)

\begin{tabular}{|c|c|c|c|}
\hline & $\begin{array}{c}\text { Part des } \\
\text { exportations } \\
\text { intra-régionales }\end{array}$ & $\begin{array}{c}\text { Part des } \\
\text { importations } \\
\text { intra-régionales }\end{array}$ & $\begin{array}{c}\text { Part du produit } \\
\text { dans le total des } \\
\text { exportations }\end{array}$ \\
\hline Tous produits confondus & $3,8 \%$ & $5,6 \%$ & $100,0 \%$ \\
\hline $\begin{array}{r}\text { Combustibles minéraux, huiles minérales, produits de leur } \\
\text { distillation }\end{array}$ & $1,4 \%$ & $10,0 \%$ & $69,6 \%$ \\
\hline Fonte, fer et acier & $3,9 \%$ & $30,1 \%$ & $4,9 \%$ \\
\hline Cuivre et ouvrages en cuivre & $0,2 \%$ & $8,4 \%$ & $3,8 \%$ \\
\hline Minerais, scories et cendres & $3,5 \%$ & $31,7 \%$ & $3,6 \%$ \\
\hline $\begin{array}{r}\text { Produits chimiques inorganiques, composés de métaux } \\
\text { précieux }\end{array}$ & $1,4 \%$ & $9,3 \%$ & $3,5 \%$ \\
\hline Perles fines ou de culture, pierres gemmes ou similaires & $0,7 \%$ & $3,3 \%$ & $2,3 \%$ \\
\hline Coton & $0,5 \%$ & $3,8 \%$ & $1,7 \%$ \\
\hline Céréales & $28,4 \%$ & $81,0 \%$ & $1,2 \%$ \\
\hline
\end{tabular}

Source : International Trade Center, 2014

Le commerce extérieur est dominé par les hydrocarbures (70\% des exportations), dont les grands consommateurs (Chine, Union Européenne, Asie du Sud-Est) sont extérieurs à la région. Dans ces conditions, l'intégration régionale n'est soutenue, de manière indirecte, que par des projets allogènes. Ainsi que l'indique Richard Pomfret (2010b, 21), «en pratique, les relations économiques internationales [entre les pays d'Asie centrale] ont été déterminées par les intérêts géopolitiques et l'unique élément économique significatif a concerné les oléoducs et les gazoducs». C'est en effet par l'exploitation des ressources minières de la région que les transformations les plus significatives ont lieu. La montée en puissance de la Chine (Raballand et Andresy, 2007) et de ses besoins en matières premières et en énergie se fait sentir partout dans la région. La Chine occupe désormais les premières places dans les flux bilatéraux d'importations et d'exportations des pays exportateurs d'énergie (Kazakhstan, Turkménistan, Ouzbékistan). Dans les pays importateurs de pétrole (Kirghizie, Tadjikistan), elle est principalement présente dans les importations, via ses produits manufacturés (Tableau 4). 
Tableau4. Principaux partenaires commerciaux des pays d'Asie centrale, 2013

\begin{tabular}{|c|c|c|c|c|c|c|c|c|c|c|}
\hline & \multicolumn{2}{|c|}{ KAZAKHSTAN } & \multicolumn{2}{|c|}{ KIRGHIZIE } & \multicolumn{2}{|c|}{ TADJIKISTAN } & \multicolumn{2}{|c|}{ TURKMÉNISTAN } & \multicolumn{2}{|c|}{ OUZBÉKISTAN } \\
\hline & Export. & Import. & Export. & Import. & Export. & Import. & Export. & Import. & Export. & Import. \\
\hline 1 & $\begin{array}{c}\text { U.E. } \\
(45 \%)\end{array}$ & $\begin{array}{l}\text { Chine } \\
(31 \%)\end{array}$ & $\begin{array}{l}\text { Kazak. } \\
(28 \%)\end{array}$ & $\begin{array}{l}\text { Chine } \\
(52 \%)\end{array}$ & $\begin{array}{c}\text { Turquie } \\
(35 \%)\end{array}$ & $\begin{array}{l}\text { Chine } \\
(42 \%)\end{array}$ & $\begin{array}{c}\text { Chine } \\
(68,5 \%)\end{array}$ & $\begin{array}{c}\text { Turquie } \\
(23 \%)\end{array}$ & $\begin{array}{l}\text { Chine } \\
(28 \%)\end{array}$ & $\begin{array}{l}\text { Chine } \\
(21 \%)\end{array}$ \\
\hline 2 & $\begin{array}{l}\text { Chine } \\
(23 \%)\end{array}$ & $\begin{array}{l}\text { U.E. } \\
(22 \%)\end{array}$ & $\begin{array}{c}\text { Ouzbék. } \\
(28 \%)\end{array}$ & $\begin{array}{l}\text { Russie } \\
(21 \%)\end{array}$ & $\begin{array}{l}\text { Iran } \\
(9 \%)\end{array}$ & $\begin{array}{l}\text { Russie } \\
(16 \%)\end{array}$ & $\begin{array}{l}\text { U.E. } \\
(9 \%)\end{array}$ & $\begin{array}{l}\text { Russie } \\
(16 \%)\end{array}$ & $\begin{array}{l}\text { Russie } \\
(20 \%)\end{array}$ & $\begin{array}{l}\text { Russie } \\
(19 \%)\end{array}$ \\
\hline 3 & $\begin{array}{c}\text { Russie } \\
(8 \%)\end{array}$ & $\begin{array}{l}\text { Russie } \\
(21 \%)\end{array}$ & $\begin{array}{l}\text { Russie } \\
(11 \%)\end{array}$ & $\begin{array}{c}\text { Kazak. } \\
(8 \%)\end{array}$ & $\begin{array}{l}\text { Chine } \\
(9 \%)\end{array}$ & $\begin{array}{l}\text { Kazak. } \\
(13 \%)\end{array}$ & $\begin{array}{c}\text { Turquie } \\
(5 \%)\end{array}$ & $\begin{array}{l}\text { U.E. } \\
(16 \%)\end{array}$ & $\begin{array}{c}\text { Corée S } \\
(16 \%)\end{array}$ & $\begin{array}{l}\text { Kazak. } \\
(13 \%)\end{array}$ \\
\hline 4 & $\begin{array}{c}\text { Canada } \\
(4 \%)\end{array}$ & $\begin{array}{c}\text { Ukraine } \\
(5 \%)\end{array}$ & $\begin{array}{c}\text { E.A.U. } \\
(7 \%)\end{array}$ & $\begin{array}{l}\text { U.E. } \\
(5 \%)\end{array}$ & $\begin{array}{l}\text { U.E. } \\
(7 \%)\end{array}$ & $\begin{array}{c}\text { Turquie } \\
(6 \%)\end{array}$ & $\begin{array}{l}\text { EAU } \\
(3 \%)\end{array}$ & $\begin{array}{c}\text { Chine } \\
(13,3 \%)\end{array}$ & $\begin{array}{l}\text { U.E. } \\
(14 \%)\end{array}$ & $\begin{array}{c}\text { Turquie } \\
(12 \%)\end{array}$ \\
\hline 5 & $\begin{array}{c}\text { Turquie } \\
(3 \%)\end{array}$ & $\begin{array}{l}\text { E.U. } \\
(3 \%)\end{array}$ & $\begin{array}{c}\text { Afghan. } \\
(6 \%)\end{array}$ & $\begin{array}{c}\text { Turquie } \\
(2 \%)\end{array}$ & $\begin{array}{c}\text { Bengla. } \\
(7 \%)\end{array}$ & $\begin{array}{l}\text { U.E. } \\
(6 \%)\end{array}$ & $\begin{array}{c}\text { Afghan. } \\
(2 \%)\end{array}$ & $\begin{array}{l}\text { EAU } \\
(7 \%)\end{array}$ & $\begin{array}{c}\text { Kazak. } \\
(11 \%)\end{array}$ & $\begin{array}{c}\text { Bengla. } \\
(8 \%)\end{array}$ \\
\hline
\end{tabular}

Source : Eurostat, calculs de l'auteur.

U.E. : Union Européenne ; E.U. : États-Unis ; E.A.U. : Émirats Arabes Unis.

\section{Les paramètres du basculement énergétique de l'Asie centrale}

Quatre paramètres principaux ont façonné l'évolution des formes de concurrence et de coopération régionales dans le secteur énergétique depuis l'indépendance : les relations nouées par les gouvernements avec les compagnies énergétiques occidentales, la construction de nouvelles routes d'exportations d'énergie, les stratégies des compagnies énergétiques russes dans la région et l'intérêt croissant de la Chine et d'autres pays asiatiques pour les ressources de la région.

Premier paramètre, le rapport aux firmes occidentales a longtemps différencié le Kazakhstan des autres pays exportateurs de la région. Elles ont été les premières à investir dans les gisements kazakhes, ce qui explique la rapidité de la reprise de la production énergétique après la chute du début des années 1990. A l'inverse, à la même époque, l'Ouzbékistan et le Turkménistan ont choisi un développement plus autocentré. La croissance de la production énergétique y a été moindre, tout en soutenant l'activité économique nationale. Dans une deuxième phase, le Kazakhstan a diversifié géographiquement ses partenariats, ce qui a permis l'irruption des entreprises chinoises dans le secteur ${ }^{2}$, tandis qu'à partir de 2007 le Turkménistan ouvrait ses gisements off-shore sur la Mer caspienne aux investisseurs étrangers après avoir accordé à la compagnie chinoise CNPC des droits de forage sur le gisement continental de Bagtyiarlyk ${ }^{3}$. La politique multi-vectorielle du Kazakhstan, associant ouverture aux entreprises occidentales, arrimage institutionnel à la Russie et développement des coopérations avec la Chine, tout en conservant des ambitions fortes en matière d'industrie nationale (Vercueil, 2014), trouve, dans le domaine énergétique, une illustration particulièrement significative. A partir de la deuxième moitié des années 2000 , ses résultats ont pu influencer l'attitude des autorités ouzbèkes et turkmènes, qui ont assoupli leur politique, jusque-là autocentrée, et se sont davantage tournées vers les entreprises étrangères.

Le deuxième paramètre - la capacité à trouver des routes de transit énergétique alternatives à la Russie est mieux partagé par les pays d'Asie centrale. Dès les années 1990, leur dépendance envers les réseaux russes (détenus par Transneft pour le pétrole et Gasprom pour le gaz) pour les approvisionnements comme pour les exportations énergétiques - la Russie jouant alors le rôle de pays de transit vers l'Europe centrale, l'Europe occidentale et les marchés lointains - est apparue comme un problème à tous les responsables politiques de la région. Cette dépendance entraînait en effet une vulnérabilité aux aléas techniques - la maintenance des réseaux n'étant pas toujours assurée de manière satisfaisante par l'opérateur russe -,

\footnotetext{
${ }^{2}$ PetroKazakbstan, compagnie canadienne initialement appelée Hurricane Hydrocarbons Ltd. et détentrice de gisements représentant $5 \%$ des réserves de pétrole du Kazakhstan, a été rachetée en 2005 par CNPCI, filiale de China Petroleum Corporation. En 2009, la compagnie nationale KazMunaiGas a acquis un tiers des actions de PetroKazakhstan.

3 "Country Analysis Brief: Turkmenistan», Energy Information Administration, january 2012 [en ligne]. Consultable sur www.eia.doe.gov [dernière consultation septembre 2014].
} 
commerciaux - les quotas et les droits de transit alloués aux compagnies pouvant varier, tout comme les taxes prélevées par l'Etat russe -, et géopolitiques - les tumultueuses relations entre la Russie et la Biélorussie puis l'Ukraine au sujet du transit du gaz vers l'Europe occidentale en témoignent -.

Les pays de la région ont développé des stratégies de diversification de leurs relations énergétiques. La réorientation des exportations de gaz du Turkménistan, dont les livraisons via le réseau de Gazprom ont chuté de $84 \%$ entre 2008 et $2011^{4}$, est une illustration récente de ce mouvement général. Après le réseau ferroviaire, intensément exploité par le Kazakhstan pour ses exportations pétrolières vers la Chine dans les années suivant l'indépendance, les infrastructures du Caspian Pipeline Consortium (CPC, 2001, pétrole), du Bakou Tbilissi Ceyban (BTC, 2005, pétrole), du Bakou Tbilissi Erzurum (BTE, 2006, gaz), du Turkménistan Chine (2009, gaz), les deux gazoducs Turkménistan Iran (1997 puis 2010) et l'oléoduc Kazakhstan Chine (dont la construction s'est étalée de 1997 à 2009) ont été développées comme autant de routes alternatives à celles héritées de l'époque soviétique. D'autres routes d'évacuation des hydrocarbures de la région sont actuellement à l'étude. Le projet de gazoduc TAPI (Turkmenistan - Afghanistan - Pakistan - Inde), actuellement à l'état d'ébauche, participe lui aussi de cette stratégie de diversification géo-énergétique, qui s'insère dans une politique de désenclavement géoéconomique largement partagée par les pays de la région (Cartes 1 et 2). A ces investissements en infrastructures s'ajoutent les coopérations techniques indispensables avec les compagnies étrangères - le plus souvent occidentales - pour explorer, évaluer, financer puis mettre en exploitation des gisements toujours plus difficiles d'accès 5 .

\section{Insérer ici les cartes 1 et 2}

La troisième clé de lecture des mouvements énergétiques en Asie centrale est la capacité des grandes entreprises russes à conduire avec succès leurs projets dans la région. Outre ses réserves en gaz - les premières du monde - et sa proximité avec le pouvoir politique russe, l'un des atouts concurrentiels favoris de Gazprom est le contrôle des réseaux de gaz nationaux dans les pays de "l'étranger proche ». Au contraire de l'Europe orientale (Moldavie et Biélorussie) et du Caucase (Arménie), ce contrôle échappait jusqu'ici totalement à Gasprom en Asie centrale. L'entreprise l'a récemment obtenu en Kirghizie par le rachat, en avril 2014, de $100 \%$ des actions de KyrgyzgazProm, filiale de la compagnie nationale Kygryzga\%, ce qui assure à la compagnie publique russe la propriété des systèmes de transmission et de distribution et le monopole des importations de gaz de la Kirghizie, qui en dépend pour $90 \%$ de sa consommation ${ }^{6}$. Gazprom est aussi présent dans l'exploration et l'exploitation en Kirghizie et au Tadjikistan et possède une entreprise conjointe avec Kaz̧MunaiGaz. (Kazakhstan) qui détient des parts dans le gisement de Karachaganak, au nord-ouest du pays. En revanche, le projet de gazoduc côtier caspien a été gelé fin 2009. Il devait doubler la partie occidentale des gazoducs turkmènes, datant de l'époque soviétique, et permettre d'assurer à Gaaprom une part significative de l'évacuation des ressources en gaz de la zone, diminuant d'autant les quantités disponibles pour les projets européens concurrents (le «couloir sud»). Ce projet demeure encore aujourd'hui dans les limbes (Carte 2).

L'autre «major» publique russe, Rosneft, est présente dans l'exploration et l'exploitation de champs pétrolifères au Kazakhstan au travers d'accords de partage de production (APP) dans les blocs d'Aday (région d'Atyrau) et Kurmangasy (mer caspienne). En Kirghizie en revanche, l'entreprise a été mise en concurrence avec des compagnies chinoises pour la rénovation de l'aéroport international de Manas, premier hub aéroportuaire du pays et ancienne base aérienne louée par l'armée américaine pour les opérations militaires de l'OTAN en Afghanistan'. Le Kazakhstan a aussi réduit en 2013 ses importations

\footnotetext{
${ }^{4}$ Cette chute de plus de $75 \%$ s'explique à la fois par des raisons techniques (explosion le 9 avril 2009 d'une branche du gazoduc Asie centrale - Centre contrôlé par Gąprom, entraînant une controverse entre les responsables turkmènes et russes sur la responsabilité de l'explosion) et économiques (baisse de la demande européenne liée à la crise économique).

${ }^{5}$ Un exemple récent de ces difficultés est le gisement pétrolier et gazier de Kachagan, situé sur la partie de la Mer noire située dans les eaux exclusives du Kazakhstan (Carte 2). Ce gisement est exploité par un consortium international piloté par l'entreprise italienne ENI.

${ }^{6}$ Les importations de gaz de la Kirghizie proviennent du Kazakhstan et de l’Ouzbékistan.

${ }^{7}$ La décision définitive n'est pas prise, pour un projet d'infrastructures dépassant le milliard de dollars. Central Asia Aero News, [en ligne], 04/07/2014. rosneft_is_not_given_landing_in_kyrgyzstan_\%E2\% $\% 0 \% 93$ _kyrgyz_airports_to_be_developed_by_china.html consultation septembre 2014]
} 
de produits pétroliers en provenance de Russie pour réaliser de l'outward processing (exportation de pétrole brut et importation de produits pétroliers) avec des raffineries chinoises, puis a adjugé à l'entreprise chinoise CNPC des droits d'exploitation dans le gisement de Kachagan pour 5 milliards de dollars8.

Dans le nucléaire, l'avance russe reste significative. L'entreprise publique Rosatom poursuit une stratégie d'intégration verticale qui couvre l'ensemble du cycle de valorisation de l'uranium ${ }^{9}$, de l'exploitation des gisements à la livraison clés en main de centrales nucléaires et au retraitement des déchets. Le 29 mai 2014, un mémorandum a été signé entre les Présidents russe et kazakhe sur la construction par Rosatom de la première centrale nucléaire de nouvelle génération du Kazakhstan, d'une capacité estimée à 1,2 GW. Des observateurs s'interrogent toutefois sur la localisation de la centrale, annoncée à la frontière nord-est du Kazakhstan, alors que le pays ne dispose pas d'un réseau électrique intégré et que ses besoins en électricité sont situés dans le sud ${ }^{10}$.

Le dernier paramètre structurant pour la région réside dans l'évolution des besoins énergétiques des grandes zones de consommation susceptibles d'offrir des débouchés aux exportateurs. La Chine et l'Union Européenne sont les deux principales, mais pas les seules. L'Inde, la Corée du Sud et le Japon sont «à portée de tubes », même si les difficultés techniques et politiques restant à surmonter pour réaliser les connexions nécessaires peuvent paraitre pour l'heure excessives. L'acteur clé reste la Chine. Les perspectives de croissance de la consommation chinoise de gaz sont à l'échelle du pays, de sa dynamique économique et de la profondeur des changements structurels qu'il entend s'imposer en réduisant sa consommation de charbon au profit du gaz. D’ores et déjà, le Turkménistan est le principal fournisseur de gaz de la Chine. Les contrats signés entre 2007 et 2012 ont abouti à la livraison de 24 milliards de mètres cubes en 2012 ( $c$. Carte 2, lignes A et B). Un accord signé en septembre 2013 prévoit de porter ce volume à 65 milliards à l'horizon 2020. La ligne $\mathrm{C}$ du gazoduc Asie centrale-Chine, parallèle aux deux précédentes, a été mise en service fin mai 2014 (Carte 2). Dès la fin de 2015, son fonctionnement à pleine capacité portera à 55 milliards de mètres cubes annuels les volumes livrables par ce réseau. D’autres accords, signés en août et septembre 2014 par la CNPC avec les compagnies ouzbèke et tadjike ont lancé la construction d'un quatrième gazoduc ( $c$. Carte 2, ligne D) qui devrait traverser l'Ouzbékistan, le Tadjikistan et la Kirghizie, pour une capacité additionnelle de 30 milliards de mètres cubes. Si les découvertes récentes de gisements gaziers dans le sud-est du Tadjikistan donnent les résultats escomptés, ces voies pourraient être doublées et les volumes exportés de la région encore augmentés significativement (Collins et White, 2013). D’ores et déjà, les volumes cumulés ${ }^{11}$ livrables à l'horizon 2018 représentent plus de deux fois ceux prévus par l'accord russo-chinois du printemps 2014, signé après dix ans de tractations (Powver of Siberia, 20182030, portant sur 38 milliards de mètres cubes annuels à partir de 2018) et parfois présenté comme le « contrat du siècle $»^{12}$. La poursuite de cette croissance dépendra de l'évolution future de la consommation énergétique chinoise, susceptible de se traduire par une hausse soutenue des besoins en importations.

\section{Demande et production énergétiques chinoises: les scénarios et leurs conséquences régionales}

Les perspectives de la consommation de gaz en Chine se situent largement au-delà des ordres de grandeur précédemment évoqués : pour limiter les émissions polluantes de ses centrales au charbon, la Chine annonce un objectif de consommation de gaz par an à l'horizon 2020, de 420 milliards de mètres

\footnotetext{
8 http://www.cacianalyst.org/publications/analytical-articles/item/12888-kazakhstan-drifts-to-china-amid-tension-withrussia.html, 08/01/2014.

${ }^{9}$ Outre l'uranium, Rosatom développe ses activités dans les minerais non ferreux et les terres rares.

${ }^{10}$ Sur ce sujet, voir par exemple http://www.breakbulk.com/breakbulk-news/general-industry/company-news/rosatom-tocomplete-kazakhstan-reactor-by-2016/ et http://www.climatenewsnetwork.net/2014/08/mystery-over-kazakh-nuclear-power-plans/

11 L'estimation réalisée par Collins et White (2013) correspond à environ 73 milliards de m3/an à l'horizon 2018.

${ }^{12}$ Les volumes envisagés par le contrat russo-chinois d'avril 2014 représentent moins de $25 \%$ des volumes annuels moyens importés par l'Union Européenne en provenance de Russie ces dernières années. Mais la perspective de la construction du gazoduc Altaï (tracé occidental du gazoduc russo-chinois) pourrait rapprocher les échéances et augmenter les capacités livrables à terme.
} 
cubes $^{13}$. Jusqu'ici, la production intérieure n'a pas suivi le rythme d'augmentation de la demande et la Chine est passée du statut d'exportateur net de gaz en 2007 à celui d'importateur net pour près de $30 \%$ de ses besoins en 2012. Quelles peuvent être les perspectives pour l'Asie centrale de la poursuite des tendances observées jusqu'ici en Chine?

Pour estimer les effets potentiels des changements observés jusqu'ici, nous proposons une simulation basée sur deux scénarios de production intérieure de gaz pour la Chine. Si la production intérieure s'accroît au rythme de la consommation à l'avenir (scénario de référence, Graphique 1) ${ }^{14}$, les accords d'ores et déjà passés avec les pays d'Asie centrale permettent en principe ${ }^{15}$ de couvrir, à pleine capacité, plus de $95 \%$ des besoins en importations en 2020. Dans ce cas, la concurrence entre l'Asie centrale et la Russie pour la fourniture des ressources nécessaires à la croissance chinoise risque de s'intensifier à l'avenir. Compte tenu des différences de coûts de production et de transport, la Russie ne partirait pas favorite pour une telle compétition. En revanche, si la croissance de la production continue au rythme observé depuis 2007 (scénario inertiel, graphique 2), les besoins en importation doubleront d'ici 2020 et les contrats, d'ores et déjà signés avec l'Asie centrale, ne permettront de fournir que $57 \%$ du total au maximum. La réalisation de ce scénario conduirait donc la Chine, selon toute probabilité, à intensifier sa pression sur les ressources gazières de l'Asie centrale d'ici 2020, tout en favorisant également une augmentation des livraisons en provenance de Russie.

\section{Graphique 1 : Importations chinoises de gaz d'Asie centrale : scénario de référence (2012-2020, milliards de mètres cubes)}

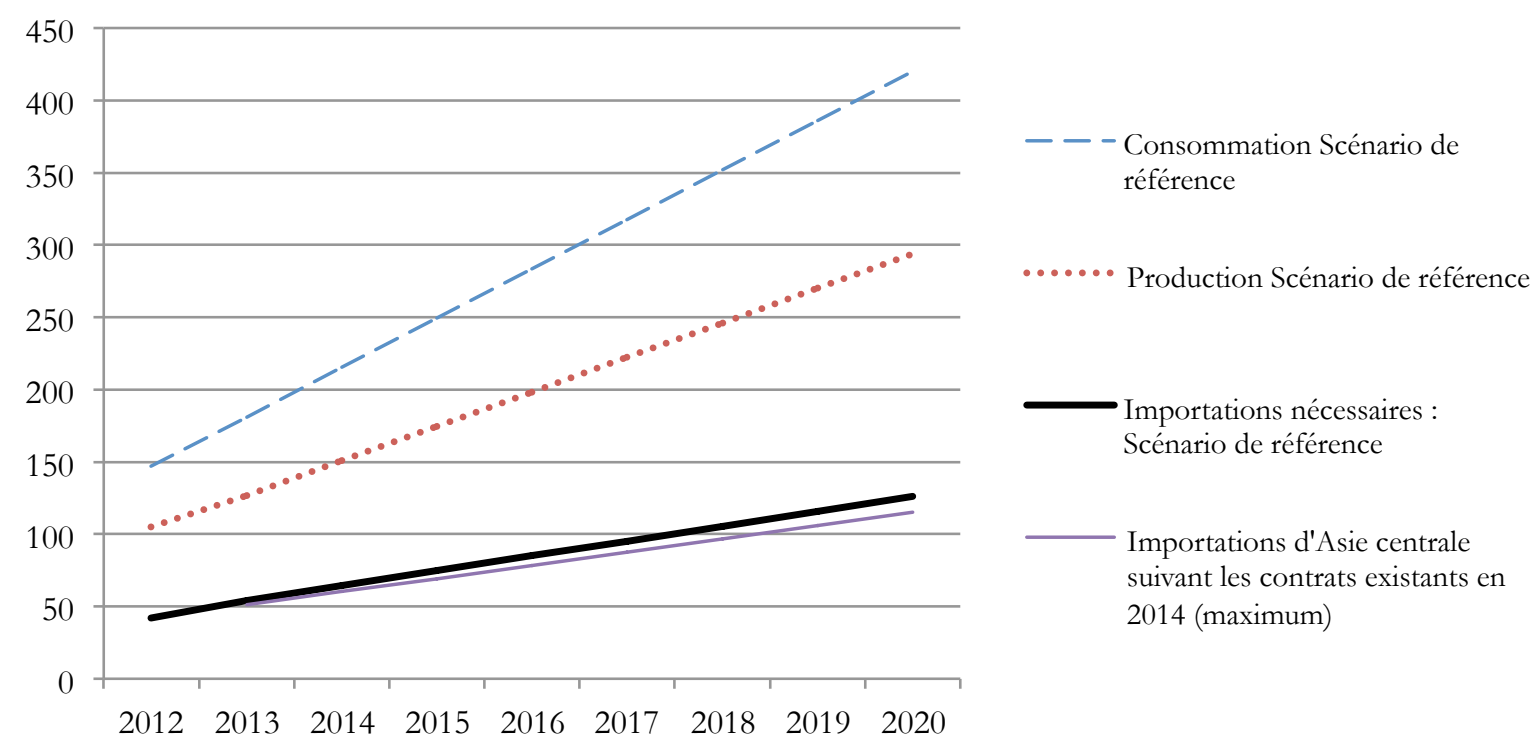

\footnotetext{
13 http://thediplomat.com/2014/05/the-significance-of-the-china-russia-gas-deal/, 24 mai 2014. Ces projections dépassent largement celles de l'agence américaine de l'énergie (EIA), qui ne prévoit pas que la Chine atteigne de tels volumes de consommation avant 2035-40. Elles supposent une accélération du rythme de croissance de la demande de gaz à $14 \%$ par an, contre $11 \%$ dans les dernières années.

${ }^{14}$ Le douzième plan énergétique (2012) prévoit par exemple que la production chinoise de gaz de schiste atteindra 65 milliards de mètres cubes en 2015 (Weidong et alii, 2014, p. 6).

${ }^{15}$ Les importations simulées dans les graphiques $3 \mathrm{a}$ et $3 \mathrm{~b}$ correspondent aux niveaux maxima envisagés par les contrats existants. Les simulations de Collins et White (2013) prévoient des flux inférieurs d'environ $30 \%$ à ces niveaux.
} 


\section{Graphique 2 : Importations chinoises de gaz d'Asie centrale : scénario inertiel (2012-2020, milliards de mètres cubes)}

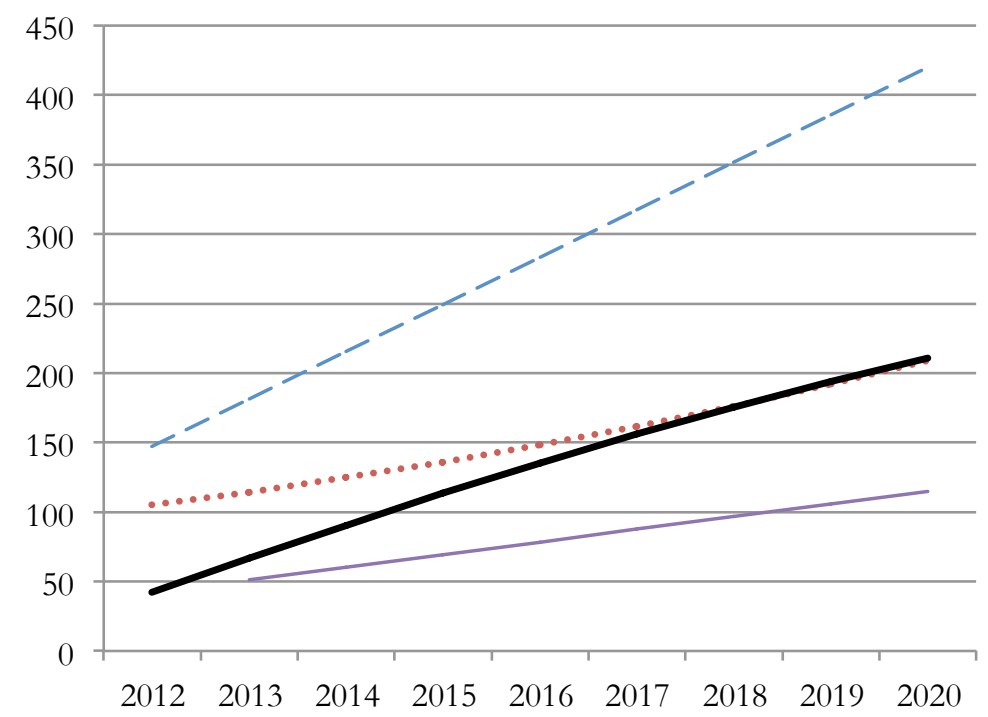

- - Consommation Scénario de référence

...... Production Scénario inertiel

Importations nécessaires : Scénario intertiel

Importations d'Asie centrale suivant les contrats existants en 2014 (maximum)

Sources : Calculs de l'auteur d'après EIA (2013)

Tout en ayant perdu le leadership en 2010 au profit de la Chine en matière d'échanges commerciaux avec l'Asie centrale ${ }^{16}$, l'Union Européenne représente un autre débouché énergétique essentiel pour la région. Ses besoins en importations de gaz en provenance d'Asie centrale sont jusqu'ici restés largement supérieurs à ceux de la Chine. Après une longue période d'hésitations, c'est le système TANAP - TAP (Trans-Anatolian Pipeline - Trans-Adriatic Pipeline) qui a finalement été choisi en juillet 2013 au détriment du projet Nabucco-Ouest $t^{77}$ pour acheminer le gaz extrait du bassin caspien - en particulier du gisement Sbab Deniz. II, au large de l'Azerbaïdjan - vers l'Europe du sud via la Turquie (Carte 2). De la sorte, le projet européen de "couloir sud", permettant à l'Europe occidentale de bénéficier d'une route d'approvisionnement en gaz qui réduit sa dépendance envers la Russie, a été relancé, le gazoduc TAP devant fournir à terme $20 \%$ des besoins énergétiques de l'UE ${ }^{18}$. Il pourrait être alimenté directement par les gisements géants du Turkménistan si le(s) projet(s) de gazoduc(s) transcaspien(s) voyai(en)t finalement le jour, surmontant les conflits de définition juridique et de partage du sous-sol de la mer caspienne entre les cinq pays riverains. La croissance des échanges entre les pays d'Asie centrale et l'Union Européenne pourrait être alors relancée, tout en appuyant la stratégie de diversification des routes d'approvisionnement énergétique engagée en Europe depuis 2007.

\section{Conclusion}

La Russie, après avoir subi un retrait brutal d'Asie centrale dans les années 1990, renoue depuis une dizaine d'années avec ses leviers d'influence traditionnels, géopolitiques et institutionnels, avec le projet d'Union Économique Eurasiatique. Elle soutient également les intérêts de ses grandes compagnies énergétiques publiques, dans le domaine pétrolier, gazier ou nucléaire. L'Union Européenne, très présente durant les années 1990, exerce moins d'attrait qu'auparavant, ses ressources financières étant contraintes et la croissance de ses marchés, en berne. Le nouvel acteur central dans la région est donc la Chine (Laruelle et Peyrouse, 2013), dont l'influence progresse via l'utilisation de leviers financiers (financements

\footnotetext{
${ }^{16}$ Les flux représentaient alors 23 milliards de dollars avec la Chine, contre 21 avec l'Union Européenne (ECFR, 2011). 17 Le projet Nabucco Ouest d'acheminement du gaz d'Asie centrale à l'Europe via la Turquie, constituait la réponse du consortium européen dirigé par l'opérateur autrichien $O M V$ à la construction d'un gazoduc russo-européen passant par la Mer noire baptisé South Stream. Plus coûteux que TAP, il a été écarté au profit de ce dernier. Depuis lors, la guerre en Ukraine et ses conséquences ont conduit le gouvernement russe à interrompre sine die la construction du gazoduc South Stream. 18 AFP, 17/12/2013.
} 
bilatéraux), logistiques (construction d'infrastructures de transport), énergétiques (oléoducs, gazoducs, barrages et contrats géants) et commerciaux (afflux de produits manufacturés et d'équipements).

Le basculement énergétique de l'Asie centrale a donc bien eu lieu. Le redimensionnement de la présence économique de la Russie que cela implique dans la région est irréversible, car il s'inscrit dans le mouvement d'intégration des économies qui la composent aux marchés régionaux et mondiaux, rationalisé par la référence à la « route de la soie » souvent mobilisée par les autorités régionales. Tout en maintenant des liens plus ou moins étroits avec la Russie, les pays de la région ont diversifié de manière décisive leurs relations vers la Chine d'un côté, l'Union Européenne (et, à un degré moindre, les ÉtatsUnis) de l'autre. Cette diversification leur offre désormais des opportunités nouvelles, au sein desquelles l'Asie (en particulier la Chine) est amenée à occuper une part croissante. Toutefois, elle n'est nullement une condition suffisante pour faire des projets correspondants de véritables leviers de développement économique régional. Contrairement à ce que prétendrait une vision irénique des effets de la montée en puissance de la Chine en Asie centrale, nous ne pensons pas que son irruption suffise à éliminer toute source de conflits dans la région. Au contraire, par son ampleur même, elle provoque des transformations et des déséquilibres régionaux qui devraient être gérés collectivement pour éviter qu'ils ne débouchent sur de nouvelles tensions économiques et géopolitiques. 


\section{BIBLIOGRAPHIE}

COLLINS G., WHITE B. (2013) Tajikistan : Pamir pipedream or new Central Asian exporter?, Oil \& Gas Journal, 05/06/2013 [en ligne], consultable sur http://www.ogj.com/articles/print/volume-111/issue-5/exploration--development/tajikistan-pamir-pipedream-or-new.html.

ECFR (2011) China Analysis : The New Great Game in Central Asia. London/Paris : European Council for Foreign Policy / Asia Centre, 13 p.

EIA (2012) 2012 Turkmenistan Country Analysis Brief U.S. Energy Intelligence Agency [en ligne], consultable sur http://www.eia.gov/countries/cab.cfm?fips=TX [dernière consultation 16/09/2014].

FREINKMAN L., POLYAKOV E., REVENCO C. (2004) Trade Performance and Regional Integration of the CIS Countries, World Bank Working Paper, n³8, June.

LARUELLE M., PEYROUSE S. (2013) Globalizing Central Asia. Geopolitics and the Challenges of Economic Development. New-York: M. E. Sharpe, 376 p.

MACIT F. (2014) Caspian Energy Outlook. Istanbul : Caspian Strategy Institute, May, 17 p.

POMFRET R. (1995) The economies of Central Asia. Pinceton, NJ : Princeton University Press, 200 p.

POMFRET R. (2006) The Central Asian economies since independence. Princeton, NJ : Princeton University Press, $256 \mathrm{p}$.

POMFRET R. (2009) Central Asia and the global economic crisis. EU-Central Asia Monitoring (EUCAM) Policy Brief $N^{\circ} 7$, Brussels : Centre of European Policy Studies (CEPS), and Madrid : Fundacion para las Relaciones Internacionales y el Dialogo Exterior (FRIDE), [en ligne], consultable sur http://aei.pitt.edu/11481/1/1880.pdf

POMFRET R. (2010a) Trade and Transport in Central Asia. Global Journal of Emerging Market Economies, 2(3), $237-$ 256.

POMFRET R. (2010b) Central Asia after two decades of independence, UNU-WIDER Working Paper \#2010-53, May.

RABALLAND G., ANDRESY A. (2007) Why should trade between Central Asia and China continue to expand ? Asia Europe Journal, 5(22), 235-252.

VERCUEIL J. (2014) : « Kazakhstan : apprendre à naviguer par gros temps », Tableau de Bord des pays d'Europe centrale et orientale et d'Eurasie, Les Études du CERI, n²210, décembre 2014, 17-22.

WEIDONG C, Xi-Min J., XIAOLAI Z. (2014) China’s Shale Gas: Current Perspectives, NAPSNet Special Reports, February 11, [en ligne], consultable sur http://nautilus.org/napsnet/napsnet-special-reports/chinas-shale-gascurrent-perspectives/ 